\title{
A Shot Number Based Approach to Performance Analysis in Table Tennis
}

\author{
by \\ Sho Tamaki ${ }^{1}$ Kazuto Yoshida ${ }^{2,3}$, Koshi Yamada ${ }^{4}$
}

The current study proposes a novel approach that improves the conventional performance analysis in table tennis by introducing the concept of frequency, or the number of shots, of each shot number. The improvements over the conventional method are as follows: better accuracy of the evaluation of skills and tactics of players, additional insights into scoring and returning skills and ease of understanding the results with a single criterion. The performance analysis of matches played at the 2012 Summer Olympics in London was conducted using the proposed method. The results showed some effects of the shot number and gender differences in table tennis. Furthermore, comparisons were made between Chinese players and players from other countries, what threw light on the skills and tactics of the Chinese players. The present findings demonstrate that the proposed method provides useful information and has some advantages over the conventional method.

Key words: table tennis, performance analysis, effectiveness.

\section{Introduction}

Table tennis is a sport in which highly refined skills and tactics are required. Players alternately and repeatedly hit a $2.7 \mathrm{~g}$ ball on a 2.75 $\times 1.525 \mathrm{~m}$ table. The ball and the court are much smaller than those employed in other racket sports. The spin, velocity and placement of the ball are controlled by players to make it difficult for opponents to successfully return it. The challenges faced by table tennis players, therefore, are to determine the optimal shot and execute it precisely, even when the ball hit by the opponent is spinning and traveling fast. In order to improve such competitive abilities, players need to analyse their performance in detail and make an effective training plan.

In recent years, performance analysis in table tennis has grown in popularity. In the International Journal of Table Tennis Science published by the International Table Tennis Federation in 2013, eight papers were categorised as 'Game Analysis', which strongly related to performance analysis, and they accounted for $22 \%$ of all papers in the journal. Performance analysis provides objective data related to players' skills and tactics and it can optimise subjective analysis. One may say that performance analysis is now one of the most important challenges in table tennis and it is conducted using various methods. Some researchers have investigated the structure of table tennis (Lanzoni et al., 2011a, 2011b, 2013; Munivrana et al., 201.5) using performance analysis on the basis of a structure model that they proposed. Some other researchers employed a stochastic model for analysing the scoring process in table tennis (Pfeiffer et al., 2010; Zhang et al., 2010). The result of the stochastic analysis

1 - Meio University, Nago City, Okinawa, Japan.

2 - Shizuoka University, Shizuoka City, Shizuoka, Japan.

3 - Sports Science and Medical Committee, International Table Tennis Federation, Lausanne, Switzerland.

4 - Table Tennis Friendship Club, Shizuoka City, Shizuoka, Japan. 
has indicated critical factors of the scoring process. The investigation of the structure or stochastic model of the scoring process is essentially important. However, elements or input variables of the models are now strictly limited as some variables, for example mechanical parameters of stroke motions or balls, physiological or psychological variables of players, are hardly measured during a match. In addition, since this type of the approach requires detailed information of a shot, the workload of data collection is always enormous. Many researchers and practical analysts have employed a simpler approach, such as the investigation of the scoring rate, losing rate or usage rate (Hao et al., 2010; Hsu, 2010; Hsu et al., 2014; Zhang et al., 2013). They implicitly assumed that we could analyse the outline of the performance by identifying the shot number (the shot number starts from the service, shot number one is for service, shot number two for service received, and so on) that has a high probability of getting or losing points, even if the details of the structure of table tennis are unknown. The main drawback of this approach is the inability to show specific skills or tactics that cause scoring or losing. Despite this drawback, such statistics are known to be useful for practitioners (Zhang et al., 2010, 2013). In addition, this approach has some advantages in terms of practical application; the required data can be collected in a short span of time and the statistics generated are easy to understand for practitioners of table tennis. The method proposed in this paper is categorised as such an approach that provides simple statistics and can be conducted in a short time.

The purpose of this study was to propose a novel approach for performance analysis in table tennis. The basic concept was similar to conventional research (Hao et al., 2010; Hsu, 2010; Hsu et al., 2014; Zhang et al., 2013). The most important and unique notion that this study proposes was to record the shot number of a scoring shot and compute the frequency or the number of shots of each shot number. By introducing the concept of frequency, statistics are more accurate and easier to understand without the need for data collection. In this paper, the advantages of the proposed method were demonstrated through the performance analysis of some of the world's elite players. In addition, a statistical comparison between Chinese players, who dominated in international competitions, and players from other countries was performed.

\section{Material and Methods}

\section{Analytical points}

The two analytical points that were considered in the proposed method were a serving rally and a shot number. In table tennis, the tactical and technical challenges strongly vary considering these factors.

A serving rally is one of the most important points to consider in table tennis analysis. The tactical and technical challenges faced by servers and receivers are different due to the special nature of service. In table tennis, a service has to rebound twice on a table, once on the server-side followed by once on the receiverside. If we could serve directly at the opponent's court as in lawn tennis, the server would have an enormous advantage. In addition, the server has to ensure collision between the racket and the ball. This table tennis rule intentionally reduces the advantage of the server. However, since table tennis players have the ability to make multiple types of services with identical motions, a service still has a great impact on a rally and its importance is often mentioned in tutorials (Geske and Muller, 2010; Molodzoff, 2008). Serving skills can influence one's scoring or losing tendency. In general, servers should take maximum advantage of a service in order to score and this is regarded as the most important challenge for them. On the other hand, for receivers, it is important to minimize the effect of a service. Thus, as described above, tactical or technical challenges vary with the serving rally.

The other point of consideration in this research is the shot number. Again, owing to the special nature of the service in table tennis, technical and tactical challenges vary with how it is received and the subsequent shots, each of which is denoted by a shot number. A service has a strong impact on the scoring or losing tendency in the early phase of rallies. If a server has good serving skills and fires a service with rotation or traveling direction difficult to anticipate, the server would get high scoring rates at the first and third shots. Therefore, the opponent would get high losing rates at the second and fourth shots. Although subsequent shots still may be affected 
by the service or shot number one, the effect of the service will gradually reduce as the number of shots increase. As the effect of services decreases, players need to face the challenge of scoring under conditions where no players have apparent advantages. Thus, as described above, it can be said that tactical or technical challenges vary with the shot number and it is one of the most important analytical points in table tennis.

\section{Inputs for the proposed method}

The inputs required for the proposed method are two types of data: a server and the shot number of the scoring shot. These two types of data per rally need to be recorded in the database. Although the scoring or losing player is not included in the inputs, they are uniquely determined through the server data and the shot number of scoring shots based on a table tennis rule that shots are hit alternately by the players.

\section{Computation of the number of shots}

At first, the number of shots for each shot number is computed. In the current study, the number of shots was defined as the 'number of shot opportunities', thus including the number of shots which missed the ball. Let us denote the number of the $i$-th shot as $S_{i}$, the server as $\mathrm{p}_{\mathrm{sv}}$, the target player under analysis as $\mathrm{p}_{\mathrm{tr}}$, the opponent player as $\mathrm{p}_{\mathrm{op}}$, the number of scoring shots as $n_{s c}$ and the function that counts the number of rallies that meet the criteria as $f_{\text {cnt }}$ (Criteria). Then, $S_{i}$ is computed by the following equations.

$$
\begin{aligned}
& \text { if } i \text { is odd, } \\
& \quad S_{i}=f_{c n t}\left(\mathrm{p}_{\mathrm{sv}}=\mathrm{p}_{\mathrm{tr}} \cap \& n_{s c} \geq i-1\right) \\
& \text { if } i \text { is even } \\
& \quad S_{i}=f_{c n t}\left(\mathrm{p}_{\mathrm{sv}}=\mathrm{p}_{\mathrm{op}} \cap \& n_{s c} \geq i-1\right)
\end{aligned}
$$

The computation of the number of shots is simple enough to perform the calculations using a spreadsheet program.

Computation of the scoring rate, losing rate and effectiveness

Next, we compute the three performance indicators: scoring rate, losing rate and effectiveness of each shot number. Let us denote the scoring player as $\mathrm{p}_{\mathrm{sc}}$, the number of the $i$-th shots scored as $n_{i}$, the number of the $i$-th shots lost as $m_{i}$, the scoring rate of the $i$-th shots as $W_{i}$, the losing rate of the $i$-th shot as $L_{i}$, the effectiveness of the $i$-th shots as $E_{i} . W_{i}, L_{i}$ and $E_{i}$, are computed by the following equations.

$$
\begin{aligned}
& W_{i}=n_{i} / S_{i} \\
& L_{i}=m_{i} / S_{i} \\
& E_{i}=W_{i}-L_{i}
\end{aligned}
$$

The scoring rate $W_{i}$ represents how good the scoring skills or tactics are at the $i$-th shot. Losing rate $L_{i}$ represents how poor the stability or returning skills are at the $i$-th shot. If a player has good defensive skills or stability, $L_{i}$ will be low. Effectiveness $E_{i}$ represents the scoring or losing tendency at the $i$-th shot. Even if a player has good offensive skills and a high $W_{i}$ value, the value of $E_{i}$ can be low when the player's shot is liable to fail and $L_{i}$ has a high value. $E_{i}$ can be regarded as contribution of the $i$-th shot to winning a match. Thus, we can see that the statistics proposed in this research are simple, intuitive and easy to understand.

\section{Comparison with the conventional method}

Conventionally, different types of statistics were used. The most commonly used statistics were a scoring rate, which is different from the one proposed in the current study, and a usage rate. Let us denote the number of scored points in a match as $p$, the number of lost points in a match as $q$, the conventional scoring rate of the $i$-th shot as $W_{i}^{\prime}$, the usage rate of the $i$-th shot as $U_{i}$. $W_{i}^{\prime}$ and $U_{i}$ were computed by the following equations.

$$
\begin{aligned}
& W_{i}^{\prime}=n_{i} /\left(n_{i}+m_{i}\right) \\
& U_{i}=\left(n_{i}+m_{i}\right) /(p+q)
\end{aligned}
$$

Conventional scoring rate $W_{i}^{\prime}$ represents the scoring tendency or bias at the $i$-th shot. If $W_{i}^{\prime}$ is high, the players' skills or tactics may be favourable to score a point at the $i$-th shot. However, it is not always the case. Let us consider the case where the target player hit a service 50 times and scored only once. If none of the services failed, $W_{1}^{\prime}$ takes the maximum value of 1.0, in spite of a rather low scoring probability. To avoid 
this type of misunderstanding, the conventional scoring rate was understood with the usage rate $U_{i}$, which represents how often the player used the $i$-th shot. There is a method to integrate the scoring rate and the usage rate into the effectiveness (Zhang et al., 2013). The conventional effectiveness of the $i$-th shot $E^{\prime}{ }_{i}$ was computed by the following equations.

$$
\begin{aligned}
E^{\prime}{ }_{i}= & -(1+\sqrt{2} / 2)+(1.5+\sqrt{2}) E o_{i} \\
& -\sqrt{2} E o_{i}{ }^{2} / 2 \\
E o_{i}= & \left(1+U_{i}\right)^{W_{i}^{\prime}-0.5}
\end{aligned}
$$

The most important advantage of the proposed method is the usage of the number of shots. In table tennis, a shot results in any one of the following three outcomes: scoring a point, losing a point or neutral, which implies that nobody scored a point. The number of neutrals was not conventionally used to understand the usage of the $i$-th shot (Equation 7) and the reason for such an approach may be related to the workload of data collection. If every number of the $i$-th shots needs to be counted manually during a match, the recording operation would become taxing and more complicated. Inaccurate methods could be selected, if they are easier to conduct and still useful. Considering this, the proposed method has little limitations. The inputs needed for the proposed method are identical to those needed for the conventional method. The player who scored and the shot number of the scoring shot are required for the conventional method, while the inputs for the proposed method are a server and the shot number of the scoring shot. In fact, the inputs for the proposed method can also be changed to the scoring player and the shot number of the scoring shot, as the data particular to the server can be obtained from the data on the scoring player and the shot number based on the rules of table tennis. Technically speaking, the inputs needed for both methods are the same. This implies that the workload required for the proposed method is identical to that required for the conventional method. The difference between the proposed method and the conventional one is only statistical accuracy. The impact of introducing the number of shots is discussed in the following sections of this paper.

\section{Match samples}

In the current study, 39 matches between male players ( 1 match played between Chinese players; 7 matches played between a Chinese player and a player from another country; and 31 matches played between players from other countries) as well as 31 matches between female players ( 1 match played between Chinese players; 6 matches played between a Chinese player and a player from another country; 27 matches played between players from other countries) were selected from the matches played at the 2012 Summer Olympics held in London. The selected matches were played by the top 50 players based on the world ranking in July 2012. The data was recorded by observing video recordings which were broadcasted on television or the internet. Written consent from the subjects was unnecessary as the matches were played in public.

\section{Statistical analysis}

1. Effectiveness, scoring rate and losing rate in table tennis

The effectiveness, scoring rate and losing rate of shot numbers, which hereinafter will be referred to as 'proposed indicators', were computed by the proposed method. The seventh and subsequent shots in a serving rally were unified into a group denoted by ' $\geq \# 7^{\prime}$ and the eighth and subsequent shots in the receiving rally were unified into a group denoted by ' $\geq \# 8^{\prime}$. When the number of shots of a particular shot number was less than five, none of the proposed indicators was computed to avoid contamination by inaccurate statistics. This kind of a process is required for all statistical analysis, not only for the proposed method. Kruskal-Wallis tests were used to assess the effect of the shot number on the proposed indicators. Wherever significant differences were observed between shot numbers, the Steel-Dwass test was used to compare the shot numbers. In addition, male players and female players were contrasted. The proposed indicators were compared with the Welch's t-test as long as the observations within two groups were normally distributed. When normality could not be assumed, the Mann-Whitney rank test was applied. Shapiro-Wilk tests were used to test the normality. Every statistical analysis was tested at a $95 \%$ confidence level. 
2. Comparison between Chinese players and players from other countries

Chinese players were contrasted with players from other countries by the proposed method. The proposed indicators were compared with the Welch's t-test as long as the observations within the two groups were normally distributed. When normality could not be assumed, the MannWhitney rank test was used. Shapiro-Wilk tests were performed to test for normality.

3. Comparison between the proposed method and the conventional method

The conventional effectiveness was computed and the linear regression coefficients between conventional effectiveness and effectiveness of the new method were determined. Pearson correlation coefficients were used to examine the relationship between the regression coefficient and the frequency of each shot number. The relationship between conventional effectiveness and the proposed method might vary with the number of shots, since conventional effectiveness does not consider frequency in its computation.

\section{Results}

\section{Effectiveness}

Figure 1a shows the distribution of effectiveness of male and female players as well as difference between genders. Table $1 \mathrm{a}$ shows a significant difference between different shot numbers for male and female players. The shot number had a significant influence on effectiveness in matches played by male $\left(\chi^{2}=147.7, p<.01\right)$ and female players $\left(\chi^{2}=86.2\right.$, $p<.01)$. Significant differences between the genders were observed in the first shot $(u=2957$. $5, \mathrm{p}<.05)$, the fourth shot $(t=-2.1, p<.05)$, the sixth shot $(u=1661.5, p<.05)$ and the seventh and subsequent shots in the serving rally $(u=$ $1582.5, p<.05)$.

\section{Scoring rate}

Figure $1 \mathrm{~b}$ shows the distribution of the scoring rate of male and female players as well as difference between genders. Table $1 \mathrm{~b}$ presents a significant difference between different shot numbers for male and female players. The shot number had a significant influence on the scoring rate in matches played by male $\left(\chi^{2}=91.4, p<.01\right)$ and female players $\left(\chi^{2}=54.3, p<.01\right)$. Also significant differences between the genders were observed in the first five shots $(\mathrm{u}=\mathrm{u}=3008.5, \mathrm{p}<.05$; $\mathrm{t}=3.3, \mathrm{p}<.01 ; \mathrm{t}=4.8, \mathrm{p}<.01 ; \mathrm{t}=3.4, \mathrm{p}<.01 ; \mathrm{u}=3138, \mathrm{p}<.01)$.

\section{Losing rate}

Figure 1c shows distribution of the losing rate of male and female players as well as difference between genders. Table 1c presents significant difference between different shot numbers for male and female players. The shot number had a significant influence on the losing rate in matches played by male $\left(\chi^{2}=306.7\right.$, $p<.01)$ and female players $\left(\chi^{2}=204.0, p<.01\right)$. Furthermore, significant differences between the genders were observed between the second and the sixth shot $(u=3014, p<.05 ; \quad u=3188.5, p<.01$; $\mathrm{t}=5.52, \mathrm{p}<.001 ; \mathrm{t}=5.26, \mathrm{p}<.01 ; \mathrm{u}=3216, \mathrm{p}<.01)$ and from the seventh and subsequent shots in serving rallies $(u=2835, p<.01)$.

\section{Comparison between Chinese players and players from other countries}

Figure 2 shows the distribution of effectiveness, scoring rate and losing rate of Chinese players and players from other countries. Chinese male players displayed higher effectiveness at the first shot $(u=299, p<.05)$, the second shot $(u=270, p<.01)$ and the fourth shot $(t=-3.5, p<.01)$, while Chinese female players presented higher effectiveness at the third shot $(u=162.5, p<.01)$, the seventh and subsequent shots in serving rallies $(u=123, p<.01)$, as well as the eighth and subsequent shots in receiving rallies $(u=-2.9, p<.01)$. Chinese male players displayed a higher scoring rate at the first shot $(\mathrm{u}=313.5, \mathrm{p}<.05)$, the second shot $(\mathrm{t}=-3.6, \mathrm{p}<.01)$, the third shot $(u=285, p<.01)$, the fourth shot $(\mathrm{t}=-$ $2.5, \mathrm{p}<.05)$ and the sixth shot $(u=260, p<.05)$, while Chinese female players presented a higher scoring rate at the second shot $(\mathrm{u}=182.5, \mathrm{p}<.05)$, the third shot $(t=-2.2, p<.05)$, the sixth shot $(\mathrm{t}=-2.4, \mathrm{p}<.05)$ and the seventh and subsequent shots in serving rallies $(u=112, p<.01)$. The significant difference of the losing rate was not observed in most shot numbers except the fourth shot by male players $(t=2.2, p<.05)$.

Comparison between the proposed method and the conventional method

Figure 3 shows the relationship between conventional effectiveness and effectiveness of the new method. A significant correlation was observed between conventional effectiveness and effectiveness of the proposed method ( $r=$ $0.88, t=60.2, p<.01)$. 


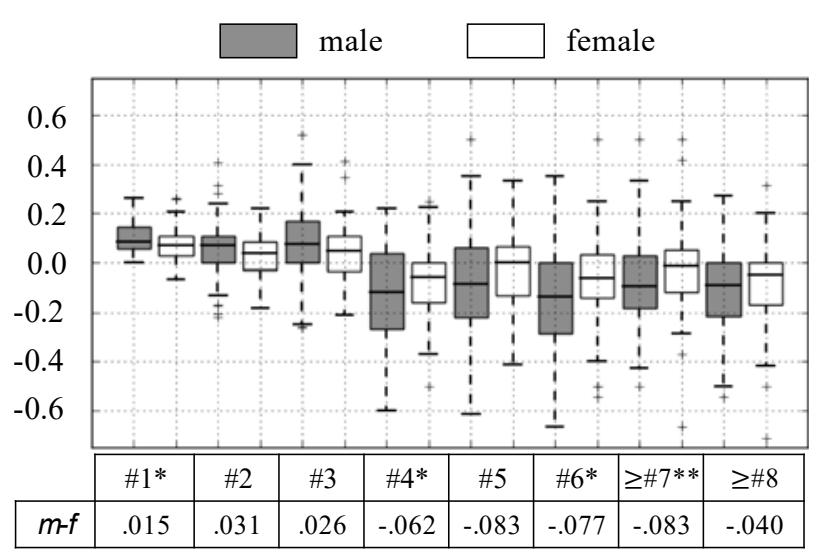

(a) Effectiveness

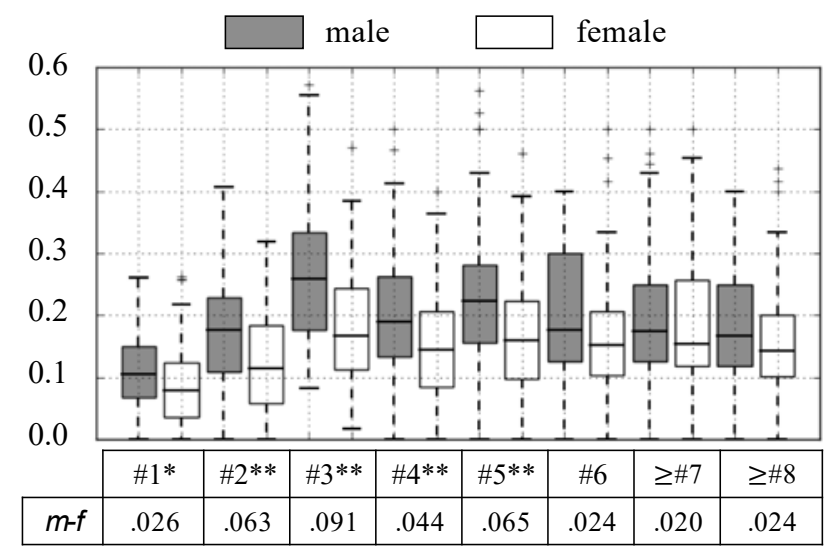

(b) Scoring rate

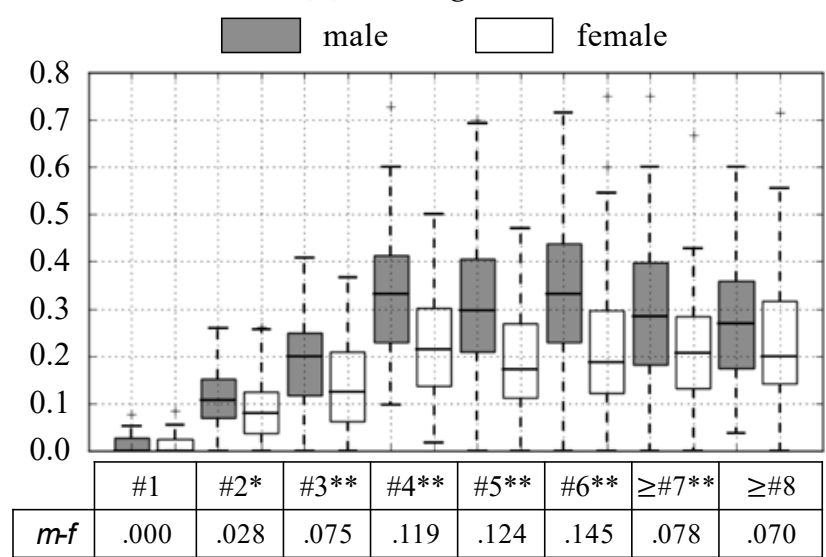

(c) Losing rate

Figure 1

Distribution of performance indicators of male and female players.

The box and whiskers denote the maximum, minimum, median, first quartile and third quartile. The cross markers denote outliers, of which distance from the box is 1.5 times as much as the length of the interquartile range. $m$-f: Difference of median between male and female players. *: Significantly different between genders $(p<.05)$. **: Significantly different between genders ( $p<.01$ ). 


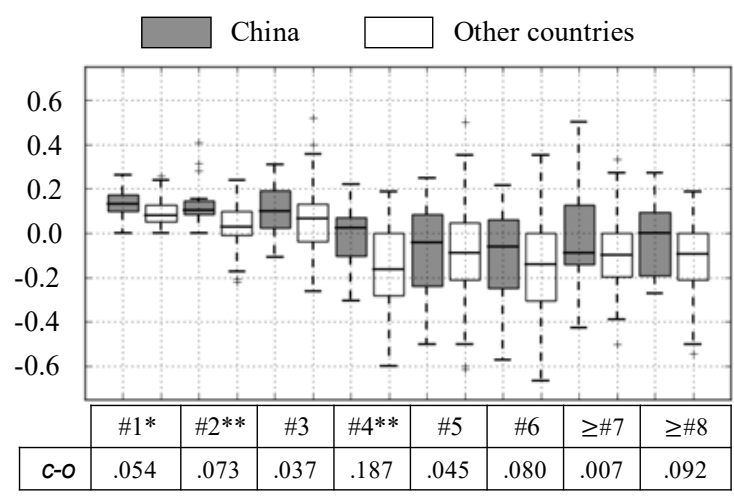

(a) Effectiveness of male players

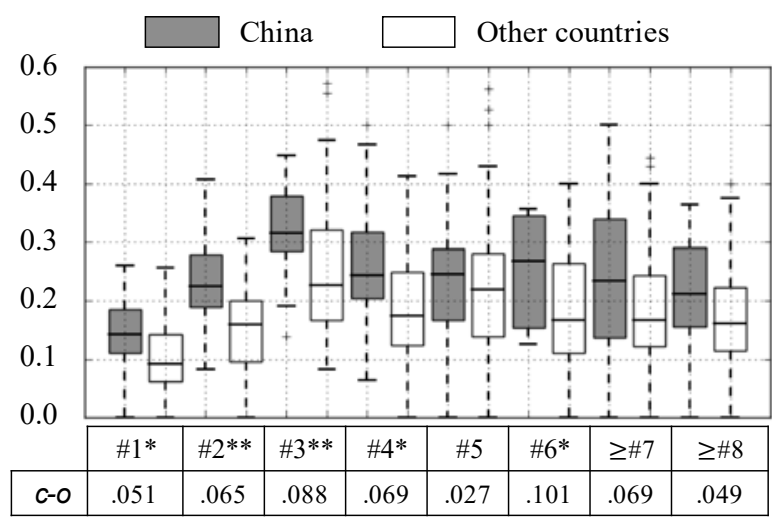

(b) Scoring rate of male players

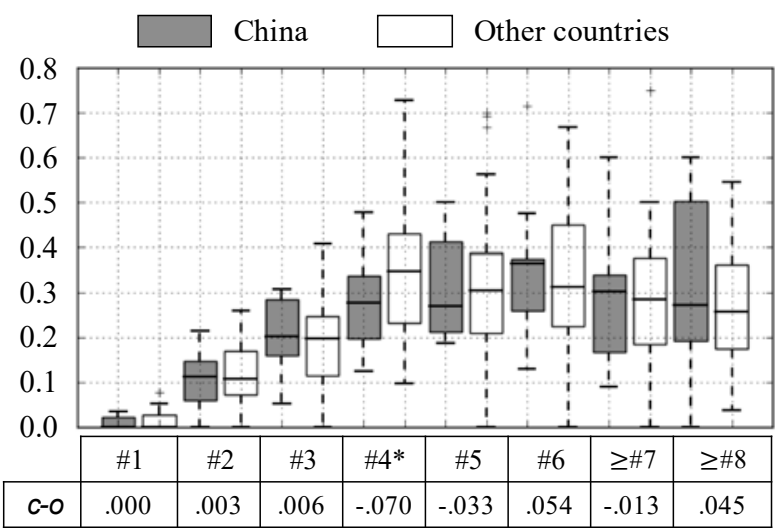

(c) Losing rate of male players

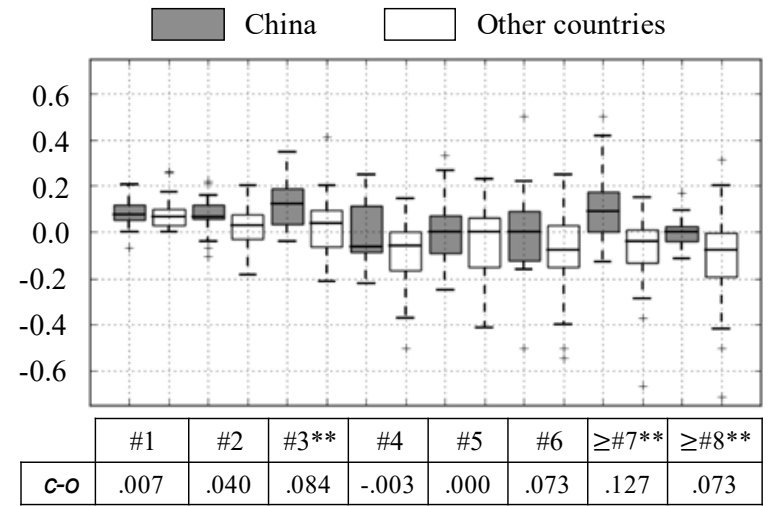

(d) Effectiveness of female players

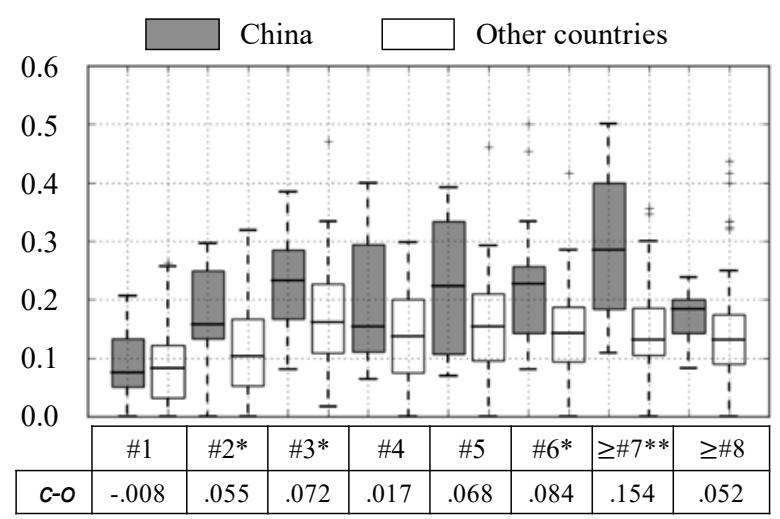

(e) Scoring rate of female players

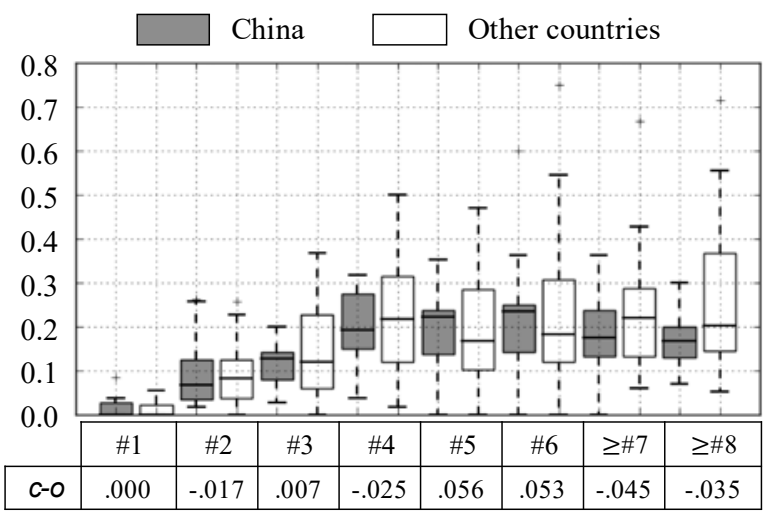

(f) Losing rate of female players

Figure 2

Distribution of performance indicators of Chinese players and the players from other countries. The box and whiskers denote the maximum, minimum, median, first quartile and third quartile. The cross markers denote outliers, of which distance from the box is 1.5 times as much as the length of the interquartile range. c-o: Difference of median between two groups. *: Significantly different between groups $(p<.05)$. ${ }^{* *}$ : Significantly different between groups $(p<.01)$. 


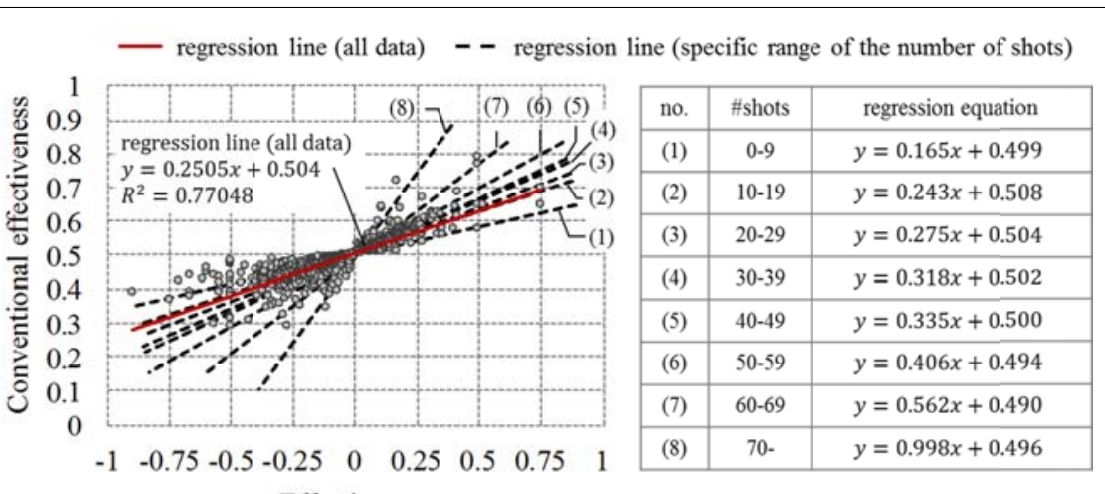

Effectiveness

Figure 3

Correlation between effectiveness of the proposed method and conventional effectiveniess.

The solid line denotes the regression line computed with all data. The dashed line denotes the regression line computed from the data of shot numbers where specific range of the number of shots was performed. The regression equation corresponding to the dashed lines is shown in the table next to the scatter plot. \#shots: the range of the number of shots.

\section{Table 1}

Multiple comparison among shot numbers

(a) Effectiveness

\begin{tabular}{|c|c|c|c|c|c|c|c|c|c|c|c|c|c|c|c|c|}
\hline & \multicolumn{8}{|c|}{ male } & \multicolumn{8}{|c|}{ female } \\
\hline & $\# 1$ & $\# 2$ & $\# 3$ & $\# 4$ & $\# 5$ & $\# 6$ & $\geq \# 7$ & $\geq \# 8$ & $\# 1$ & $\# 2$ & $\# 3$ & $\# 4$ & $\# 5$ & \#6 & $\geq \# 7$ & $\geq \# 8$ \\
\hline$\# 1$ & & n. s. & n. s. & $>* *$ & $>* *$ & $>* *$ & $>* *$ & $>* *$ & & n. s. & n. s. & $>* *$ & $>* *$ & $>* *$ & $>* *$ & $>* *$ \\
\hline$\# 2$ & n. s. & & n. s. & $>* *$ & $>* *$ & $>* *$ & $>* *$ & $>* *$ & n. s. & & n. s. & $>* *$ & n. s. & $>* *$ & $>*$ & $>* *$ \\
\hline$\# 3$ & n. s. & n. s. & & $>* *$ & $>* *$ & $>* *$ & $>* *$ & $>* *$ & n. s. & n. s. & & $>* *$ & n. s. & $>* *$ & $>*$ & $>* *$ \\
\hline$\# 4$ & $<* *$ & $<* *$ & $<* *$ & & n. s. & n. s. & n. s. & n. s. & $<* *$ & $<* *$ & $<* *$ & & n. s. & n. s. & n. s. & n. s. \\
\hline$\# 5$ & $<* *$ & $<* *$ & $<* *$ & n. s. & & n. s. & n. s. & n. s. & $<* *$ & n. s. & n. s. & n. s. & & n. s. & n. s. & n. s. \\
\hline$\# 6$ & $<* *$ & $<* *$ & $<* *$ & n. s. & n. s. & & n. s. & n. s. & $<* *$ & $<* *$ & $<* *$ & n. s. & n. s. & & n. s. & n. s. \\
\hline$\geq \# 7$ & $<* *$ & $<* *$ & $<* *$ & n. s. & n. s. & n. s. & & n. s. & $<* *$ & $<*$ & $<*$ & n. s. & n. s. & n. s. & & n. s. \\
\hline$\geq \# 8$ & $<* *$ & $<* *$ & $<* *$ & n. s. & n. s. & n. s. & n. s. & & $<* *$ & $<* *$ & $<* *$ & n. s. & n. s. & n. s. & n. s. & \\
\hline
\end{tabular}

(b) Scoring rate

\begin{tabular}{|c|c|c|c|c|c|c|c|c|c|c|c|c|c|c|c|c|}
\hline & \multicolumn{8}{|c|}{ male } & \multicolumn{8}{|c|}{ female } \\
\hline & $\# 1$ & $\# 2$ & $\# 3$ & $\# 4$ & $\# 5$ & $\# 6$ & $\geq \# 7$ & $\geq \# 8$ & $\# 1$ & $\# 2$ & $\# 3$ & $\# 4$ & $\# 5$ & $\# 6$ & $\geq \# 7$ & $\geq \# 8$ \\
\hline$\# 1$ & & $<* *$ & $<* *$ & $<* *$ & $<* *$ & $<* *$ & $<* *$ & $<* *$ & & n. s. & $<* *$ & $<* *$ & $<* *$ & $<* *$ & $<* *$ & $<* *$ \\
\hline$\# 2$ & $>* *$ & & $<* *$ & n. s. & $<*$ & n. s. & n. s. & n. s. & n. s. & & $>* *$ & n. s. & n. s. & n. s. & $>*$ & n. s. \\
\hline$\# 3$ & $>* *$ & $>* *$ & & $>*$ & n. s. & $>*$ & $>* *$ & $>* *$ & $>* *$ & $<* *$ & & n. s. & n. $s$. & n. s. & n. s. & n. s. \\
\hline$\# 4$ & $>* *$ & n. s. & $<* *$ & & n. s. & n. s. & n. s. & n. s. & $>* *$ & n. s. & n. $s$. & & n. s. & n. s. & n. s. & n. s. \\
\hline$\# 5$ & $>* *$ & $>*$ & n. s. & n. s. & & n. s. & n. s. & n. s. & $>* *$ & n. s. & n. s. & n. s. & & n. s. & n. s. & n. s. \\
\hline$\# 6$ & $>* *$ & n. s. & $<* *$ & n. s. & n. s. & & n. s. & n. s. & $>* *$ & n. s. & n. s. & n. s. & n. s. & & n. s. & n. $s$. \\
\hline$\geq \# 7$ & $>* *$ & n. s. & $<* *$ & n. s. & n. s. & n. s. & & n. s. & $>* *$ & $<*$ & n. s. & n. s. & n. s. & n. s. & & n. s. \\
\hline$\geq \# 8$ & $>* *$ & n. s. & $<* *$ & n. s. & n. s. & n. s. & n. s. & & $>* *$ & n. s. & n. s. & n. s. & n. s. & n. s. & n. s. & \\
\hline
\end{tabular}

(c) Losing rate

\begin{tabular}{|c|c|c|c|c|c|c|c|c|c|c|c|c|c|c|c|c|}
\hline & \multicolumn{8}{|c|}{ male } & \multicolumn{8}{|c|}{ female } \\
\hline & $\# 1$ & $\# 2$ & $\# 3$ & $\# 4$ & $\# 5$ & $\# 6$ & $\geq \# 7$ & $\geq \# 8$ & $\# 1$ & $\# 2$ & $\# 3$ & $\# 4$ & $\# 5$ & $\# 6$ & $\geq \# 7$ & $\geq \# 8$ \\
\hline$\# 1$ & & $<* *$ & $<* *$ & $<* *$ & $<* *$ & $<* *$ & $<* *$ & $<* *$ & & $<* *$ & $<* *$ & $<* *$ & $<* *$ & $<* *$ & $<* *$ & $<* *$ \\
\hline \#2 & $>* *$ & & $<* *$ & $<* *$ & $<* *$ & $<* *$ & $<* *$ & $<* *$ & $>* *$ & & $<*$ & $<* *$ & $<* *$ & $<* *$ & $<* *$ & $<* *$ \\
\hline$\# 3$ & $>* *$ & $>* *$ & & $<* *$ & $<* *$ & $<* *$ & $<* *$ & $<*$ & $>* *$ & $>*$ & & $<* *$ & n. s. & $<*$ & $<* *$ & $<* *$ \\
\hline$\# 4$ & $>* *$ & $>* *$ & $>* *$ & & n. s. & n. s. & n. s. & n. s. & $>* *$ & $>* *$ & $>* *$ & & n. s. & n. s. & n. s. & n. s. \\
\hline$\# 5$ & $>* *$ & $>* *$ & $>* *$ & n. s. & & n. s. & n. s. & n. s. & $>* *$ & $>* *$ & n. s. & n. s. & & n. s. & n. s. & n. s. \\
\hline$\# 6$ & $>* *$ & $>* *$ & $>* *$ & n. s. & n. s. & & n. s. & n. s. & $>* *$ & $>* *$ & $>*$ & n. s. & n. s. & & n. s. & n. s. \\
\hline$\geq \# 7$ & $>* *$ & $>* *$ & $>* *$ & n. s. & n. s. & n. s. & & n. s. & $>* *$ & $>* *$ & $>* *$ & n. s. & n. s. & n. s. & & n. s. \\
\hline$\geq \# 8$ & $>* *$ & $>* *$ & $>*$ & n. s. & n.s. & n. s. & n. s. & & $>* *$ & $>* *$ & $>* *$ & n. s. & n.s. & n. s. & n. s. & \\
\hline
\end{tabular}

$<,>$ : " $<$ " denotes that the left shot number is significantly less than the upper shot number, and " $>$ " denotes the that the left shot number is significantly greater than the upper shot number. ${ }^{*} p<0,05:{ }^{* *} p<0,01$ : . 


\begin{tabular}{|c|c|c|c|c|c|c|c|}
\hline \multicolumn{8}{|c|}{$\begin{array}{c}\text { Table } 2 \\
\text { An example of two data sets with identical effectiveness of the proposed method } \\
\text { and different conventional effectiveness }\end{array}$} \\
\hline \#shot & W & $\mathrm{L}$ & \#shots & all scoring & all losing & $\mathrm{E}$ & $\mathrm{E}^{\prime}$ \\
\hline A & 7 & 0 & 37 & 28 & 44 & 0.189 & 0.570 \\
\hline $\mathrm{B}$ & 9 & 6 & 16 & 41 & 34 & 0.188 & 0.527 \\
\hline \multicolumn{8}{|c|}{$\begin{array}{l}\text { \#shot: shot number. W: number of scoring points. L: number of losing points. } \\
\text { \#shots: frequency of the shot number. all scoring: number of points scored in the } \\
\text { match. all losing: number of points lost in the match. E: effectiveness of the new } \\
\text { method. E': conventional effectiveness. }\end{array}$} \\
\hline
\end{tabular}

The dashed lines in the scatter plot and the table next to the scatter plot in Figure 3 show the regression equations that were computed from the data where a specific range of the number of shots was performed. The correlation between the regression coefficient and the number of shots was significant $(r=0.69, t=29.7, p<.01)$. Thus, it can be stated that the relationship between conventional effectiveness and effectiveness of the new method varied with regard to the number of shots.

\section{Discussion}

\section{Effectiveness, scoring rate, and losing rate in} table tennis

The results show characteristics of shot numbers in table tennis rallies. The effectiveness of the first three shots was identical and higher than this of the other shot numbers. Effectiveness can be analysed on the basis of the scoring and losing rates. The common characteristic of the first three shots was a low losing rate. Although the first two shots had a low probability of scoring, the effectiveness was high due to the low losing rate. The third shot had a relatively low losing rate and, moreover, a high scoring rate especially in matches played by male players. This suggests how table tennis players take advantages of the service. It is easier to score at the third shot with a service, even if one hardly scores directly with a service itself. However, the effect of the service or the advantages of servers do not persist for long in a rally. According to Table 1, few differences were found among shot numbers that were greater than three.

Male players hit a ball with a higher probability of scoring than female players. This result is intuitively understandable because, generally, male players can hit a ball with stronger force than female players. Keeping this fact in mind, the scoring rate of the first shot can be considered remarkable, as the strength of male players hardly contributed to the velocity of services based on the rule of table tennis, i.e. services have to rebound twice. These results

imply two possible explanations: male players have good serving skills, which female players rarely have, or male players offensively hit a ball at the second shot with a risk of failure. Although the current study cannot provide more details, some insights into table tennis can be gained through the proposed method.

Comparison between Chinese players and the players from other countries

The results suggest that male Chinese players had better skills at receiving rallies than players from other countries (Figure 2a). The difference in the second and the fourth shot, namely the first two shots in a receiving rally, were most noticeable. Let us assume 10 receiving rallies were performed and a player hit the ball 10 times at the second and fourth shots, which roughly corresponds to the receiving rallies in a game (a game is a part of a match in table tennis, a match consists of an odd number of games). In this case, the difference in the effectiveness of the second and fourth shots, 0.073 and 0.187 , is 
equivalent to the difference of 0.7 and 1.9 points, respectively. The fourth shot showed the biggest difference and this could determine the winner of a game. Although the difference in the second shot was smaller than that of the fourth shot, the effectiveness of the fourth shot can be strongly influenced by the skills and tactics used at the second shot. As a result, the topmost priority for the players from other countries to win against Chinese players is to minimize the difference in receiving rallies. Since the differences in effectiveness were derived from both the scoring rate as well as the losing rate, their scoring skills and returning skills need to improve. Improving scoring skills at the first shot or service will be the next challenge.

The results suggest that female Chinese players had better scoring skills at the third shot and long rallies (Figures $2 \mathrm{~d}$ and 2e). These differences were mostly derived from the scoring rate (Figures $2 \mathrm{e}$ and $2 \mathrm{f}$ ). The scoring rate of the seventh and subsequent shots, especially in serving rallies, was high and close to the third shot of male Chinese players. This result is noteworthy because, as stated earlier, high shot numbers in a rally result in conditions where none of players has any advantage. These results show that female Chinese players had outstanding scoring skills in long rallies, which might be related to the high scoring rate at the third shot.

Thus, it was shown that performances of table tennis players may be successfully compared on the basis of the proposed method.

\section{Comparison between the proposed method and the conventional method}

The proposed method is more suitable for analysis and aims to evaluate players' skills and tactics. A strong correlation between the effectiveness of the new method and conventional effectiveness was observed with certainty. However, it was also observed that the relationship between them considerably varied with the number of shots. That is, when a shot number with lower frequency was executed, lower conventional effectiveness was computed, even if the effectiveness of the new method remained the same. Considering the example shown in Table 2, the difference in conventional effectiveness was 0.043, which is a two-level difference in the conventional evaluation criteria (Zhang et al., 2013) consisting of four levels in total. However, the difference in effectiveness of the new method was 0.001 and it would have made a difference of only 0.1 points even if 100 rallies had been performed. This difference occurs when we compare data sets which contain values for the number of shots that are substantially different from each other. The conventional effectiveness becomes considerably low when the sum of the number of scoring and losing points is small. This characteristic is statistically undesirable for the evaluation of skills and tactics because effectiveness is independent of the sum of the number of scoring and losing points. This result suggests that conventional effectiveness was designed to evaluate the actual volume of effect caused by each shot number in a match rather than to evaluate the skills or tactics. When the purpose of the analysis is to evaluate the players' skills or tactics, the proposed new method should be used instead of the conventional one. Even when the purpose of the analysis is to evaluate the actual effect caused by each shot number in a match, the effectiveness of the proposed method considering the number of shots might be more accurate and suitable than indicators that do not include the number of shots.

The analysis of effectiveness can be obtained through the proposed method. The scoring rate is related to scoring skills and the losing rate is connected with the stability or defensive skills. When we find high effectiveness for a certain shot number, we are able to determine if it was derived from a high scoring rate or a low losing rate. Conventionally, the analysis of the effectiveness cannot be performed.

The proposed method is simpler and easier to understand than the conventional one. The effectiveness proposed in the current study can be computed as a unit of points by multiplying the assumed number of shots, as shown in an earlier subsection. In contrast, conventional effectiveness cannot be derived without comparing the distribution of multiple matches. Even when some difference between two data sets was seen, the impact of the difference on a match was hardly perceptible. In addition, it is essentially impossible to compare different shot numbers, since their number of shots are substantially different. We need to understand the conventional effectiveness with multiple criteria for each shot 
number. As a result of the above discussion, it can be said that the proposed method has many advantages over the conventional method, and is useful for performance analysis in table tennis.

\section{Limitations of the proposed method}

The proposed method cannot determine the dominant factor of a high or low scoring / losing rate, while it provides more specific information than the conventional one does. For example, a high scoring rate in the third shot seems to indicate that the player has good scoring skill at the third shot. However, there are also some other possibilities, such as that for example the first shot was good and it created an opportunity to score at the third shot or the opponent's skill of the second shot was poor. The proposed method provides no information to discriminate between them.

To make the statistics reliable, the lower bound of the number of shots needs to be decided arbitrary. When the number of shots is extremely small, the effectiveness, scoring rate or losing rate may be extremely high or low. Although this problem is common to many other statistical methods, it can induce a difficult discussion in the practical scenario where the proposed method is used. Many additional trials may be required to find the optimal lower bound of the number of shots to calculate the proposed statistics in a reliable way. It is a limitation of this study and an issue to be considered in future research.

\section{Conclusion}

A novel method for performance analysis in table tennis was proposed. The proposed method improved the conventional analysis by introducing the number of shots for each shot number, which was computed on the basis of the shot number of a scoring shot. The advantages of the proposed method over the conventional method may be summarised as follows:

(1) Skills or tactics are more accurately evaluated than in the conventional method.

(2) Scoring skills and returning skills can be evaluated separately, whereas these insights cannot be gained conventionally.

(3) The results can be understood easily as they can be converted into a unit of points.

(4) The results can be understood with a single criterion, whereas conventional analysis requires multiple criteria for each shot number.

The performance analysis of matches played in the 2012 London Olympics was conducted on the basis of the proposed method. The results of the analysis clarified the scoring or losing bias caused by shot numbers and gender difference in table tennis. Chinese players and players from other countries were then compared. The results showed that skills and tactics in receiving rallies were significantly different in matches played by male players, and scoring skills and tactics in long rallies were significantly different in matches played by female players. The present findings demonstrate that the proposed method provides useful information and has advantages over the conventional method.

\section{References}

Geske KM, Muller J. Table tennis tactics. United Kingdom: Meyer \& Meyer Sport, 71-77; 2010

Hao Z, Tian Z, Hao Y, Song J. Analysis on Technique and Tactics of Lin Ma and Hao Wang in the Men's Single Table Tennis Final in the 29th Olympic Games. International Journal of Table Tennis Science, 2010; 6: $74-78$

Hsu MH. A Study on the Technical Analysis and Attack-Defense Performance of Men's Top Four Single Players in 2008 Olympic Games. International Journal of Table Tennis Science, 2010; 6: 248-260

Hsu MH, Chen YF, Wang SC. Offense - Defense Mode Analysis of the World Top Male Table Tennis Player A Case Study by Chuang Chi-Yuan Who Participated in 2012 London Olympic Male Single Games. Journal of Science and Innovation, 2014; 4(2): 41-50

Lanzoni IM, Michele RD, Merni F. Performance indicators in table tennis: a review of the literature. Proceedings of 
the $12^{\text {th }}$ ITTF Sports Science Congress, 2011; 71-75

Lanzoni IM, Michele RD, Merni F. Reliability of selected table tennis performance indicators. Proceedings of the $12^{\text {th }}$ ITTF Sports Science Congress, 2011; 62-65

Lanzoni IM, Michele RD, Merni F. A notational analysis of shot characteristics in top-level table tennis players. European Journal of Sport Science, 2013; 14(4): 309-317

Molodzoff P. ITTF Advanced Coaching Manual. Lausenne: International Table Tennis Federation, 105-107; 2008

Munivrana G, Petrinovic LZ, Kondric M. Structural Analysis of Technical-Tactical Elements in Table Tennis and their Role in Different Playing Zones. Journal of Human Kinetics, 2015; 47: 197-214

Pfeiffer M, Zhang H, Hohmann A. A Markov Chain Model of Elite Table Tennis Competition. International Journal of Sports Science and Coaching, 2010; 5(2): 205-222

Zhang H, Yu L, Hu J. Computer-aided Game Analysis of Net Sports in Preparation of Chinese Teams for Beijing Olympics. International Journal of Computer Science in Sport, 2010; 9(3): 53-69

Zhang H, Liu W, Hu JJ, Liu RZ. Evaluation of elite table tennis players' technique effectiveness. J Sport Sci, 2013; 31(14): 1526-1534

\section{Corresponding author:}

\section{Sho Tamaki}

Meio University

1220-1 Biimata, Nago City, Okinawa, Japan

Phone: +81 980511522

E-mail: s.tamaki@meio-u.ac.jp 